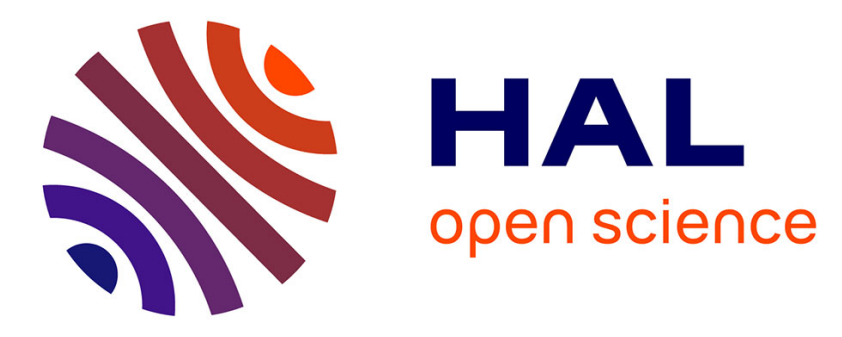

\title{
Enhancing Decision-Making in New Product Development: Forecasting technologies revenues using a Multidimensional Neural Network
}

\author{
Marie Saade, Maroun Jneid, Imad Saleh
}

\section{- To cite this version:}

Marie Saade, Maroun Jneid, Imad Saleh. Enhancing Decision-Making in New Product Development: Forecasting technologies revenues using a Multidimensional Neural Network. EMCIS 202017th European Mediterranean \& Middle Eastern Conference on Information Systems, 2020, Dubai, United Arab Emirates. hal-03017387

\section{HAL Id: hal-03017387 \\ https://hal.science/hal-03017387}

Submitted on 20 Nov 2020

HAL is a multi-disciplinary open access archive for the deposit and dissemination of scientific research documents, whether they are published or not. The documents may come from teaching and research institutions in France or abroad, or from public or private research centers.
L'archive ouverte pluridisciplinaire HAL, est destinée au dépôt et à la diffusion de documents scientifiques de niveau recherche, publiés ou non, émanant des établissements d'enseignement et de recherche français ou étrangers, des laboratoires publics ou privés. 


\title{
Enhancing Decision-Making in New Product Development: Forecasting technologies revenues using a Multidimensional Neural Network
}

\author{
Marie SAADE ${ }^{1, *[0000-0003-0520-585 x]}$, Maroun JNEID $^{2}, \operatorname{Imad}^{\text {SALEH }}{ }^{1}$ \\ ${ }^{1}$ Laboratoire Paragraphe (EA 349), Université Paris 8 Vincennes-Saint-Denis, \\ Saint-Denis, France \\ marie.karameetud.univ-paris8.fr, imad.saleh@univ-paris8.fr \\ 2 TICKET Lab., Antonine University, Hadat-Baabda, Lebanon \\ maroun.jneid@ua.edu. lb
}

* Corresponding author

E-mail: marie.karam@etud.univ-paris8.fr (MS)

\begin{abstract}
Aiming to retain their position in the marketplace, organizations are constantly enhancing research and development-based digital innovation activities in order to constantly develop new products and deploy new technologies. However, innovative trends and products are prone to failure, leading to undesired repercussions. In addition, when evaluating a product lifecycle, many decision-makers confront unprecedented challenges related to the estimation of potential disruptive innovation. To address this gap and to tackle the opportunities of digitalization, we conduct quantitative study to investigate the usage of research and development activities that can represent a main economic driver for new product/service development. A new approach for predicting innovative technology-based product success is proposed using Neural Networks models and based on the analysis of patents, publications and technologies revenues which are considered major key performance indicators in measuring technology-based product power. The proposed methodology consists of two main steps: forecasting patents and publications growths separately for a specific candidate technology using a common predictive Neural Network regression model, then integrating the results into a Multidimensional Neural Network classifier model in order to predict future revenue growth for this candidate technology. The present methodology is applied using two different types of Neural Networks for comparison purpose: "Wide and Deep Neural Networks" and "Recurrent Neural Networks". Consequently, addressing this estimation represents a decision support and a crucial prerequisite step before proceeding with investments, where organizations can improve decision making in innovative technology-based product/service development. The findings show that the Recurrent Neural Networks models achieve higher prediction accuracy, and outperform the Wide and Deep Neural Networks, proving to be a more reliable model that can enhance digital innovation development.
\end{abstract}

Keywords: Innovative product development. Forecasting product revenues. Neural Networks. Decision support. 


\section{Introduction}

Nowadays, with the intention to increase financial revenues and to determine competitiveness in the market, organizations are constantly enhancing research and development-based digital innovation activities which represent a main driver for new product/service development [1]. For instance, Jneid and Saleh stated in their study that innovation represents the main component that contributes to the success of new start-ups encountering a competitive environment [2]. However, despite the fact that organizations are constantly developing new products while increasing R\&D investments and deploying new technologies, innovative trends are prone to failure, leading to undesired repercussions [3]. Furthermore, when evaluating a product lifecycle, the decision-makers of the organization first need to consider and estimate the potential disruptive innovation, in order to assess when a product is threatened to enter the decline phase [4]. For example, Apple with its innovative iPhone series and Samsung with its Android operating system forced Nokia phones to enter the decline phase of their life-cycle in 2007 [5].

Nevertheless, while innovation disruptiveness can threaten the existing product market performance, the adoption of this disruptive technology may improve and lead to a new product development [6].

The main problematic of the current study is to explore how disruptive innovation can be estimated quantitatively in an early stage, and therefore to examine how digital innovation development can be enhanced by forecasting new technology-based product success by applying a convenient predictive technique that can support the decision-making in the organizations.

Several previous studies proposed different methodologies to identify a potential disruptive innovation in order to improve efficiency during new product development process. Nagy, Schuessler and Dubinsky [7] suggested a qualitative study that can determine if a new candidate technology would be a potential disruptive innovation. This method highlights the importance of comparing the technical standards, the functionality and the ownership of this new candidate innovation with the existing technology currently used in the organization. In addition, Momeni and Rost [8] proposed a systematic tool that can analyze quantitatively the relationship between technological disruptiveness and highly cited patents. It is based on patentdevelopment path, topic-modeling used for patent-citation analysis and k-core analysis to classify different subgroups of each technology. A quantitative study of a visualization bibliometric analysis has been elaborated as well to explore potential disruptive innovation [9].

However, each of those methodologies has limitations. For instance, the aforementioned qualitative procedure often rely on subjective judgments of decisionmaking experts, which may influence results [9]. The patent-development path solution and the bibliometrics-based analysis cover only single indicators, patents and bibliometrics dimensions respectively.

Consequently, to address this gap, the technologies-based products development process can be monitored by analyzing historical data of the related R\&D activities and products revenues. Specifically, patents, publications and revenues are considered major key performance indicators and effective dimensions in measuring technologybased product power. In this regard, we propose a new methodology based on two main steps: forecasting patents and publications growths separately for a specific candidate technology using a common predictive Neural Network regression, then integrating the results into a Multi-dimensional Neural Network classifier in order to predict the future revenue-volume for this candidate technology. The present methodology is applied using two different types of Neural Network models for comparison purpose: "Wide and Deep Neural Networks" and "Recurrent Neural Networks".

Consequently, addressing this estimation represents a decision support in technologybased product/service development and a prerequisite step before proceeding with investments. 
The present study represents a proof of concept where it focuses on forecasting the patents, publications and revenues dimensions growth for a single candidate technology, noting that when this approach is to be applied on a real case study on organizational level, it can involve both a new technology representing a potential disruptive innovation, and an existing technology currently used in the organization, for comparison purposes.

In addition, given the fact that a single product may embed several combined technologies (such as a drone that can be composed of 3D mapping technology, Infrared cameras, etc.), and since patents and publications data are technology-bound whereas revenues data are product-bound, the current study focuses on a specific type of product which is based on one major trending technology.

The remainder of this paper is structured as following: Section 2 presents the related work on the patent, publication and revenues-based forecasting methods. Section 3 presents the research design and the proposed methodology. The experimental setting of the present study and the results are described in section 4 . Section 5 concludes the work and points to the study limitations and to future research directions.

\section{$2 \quad$ Literature Review}

R\&D-based digital innovation activities represent a main driver for new product/service development. Patents data play a significant role in forecasting the success of trending technologies as justified in the paper "Predicting Technology Success based on Patent Data, using a Wide and Deep Neural Network and a Recurrent Neural Network" [10]. Publications data can be used as well to measure technological and scientific capabilities [11]. Furthermore, patents and publications data are considered among the most important dimensions that have potential benefits and reflection into the knowledge-based economy [12, 13].

Patent and Publication-based technology forecasting methods: Growth curves, scenario planning and analogies technology forecasting tools have been used based on patent and bibliometrics analysis, while focusing on food safety, fuel cell and optical storage technologies [14]. Moreover, patent analysis, bibliometric analysis and the technology roadmapping method have been combined to visualize and predict the future development of the Nanogenerator technology in China [15].

Revenue forecasting studies: Different revenue forecasting techniques are used to evaluate the product level and thus to support planning and decision making. An efficient solution based on three different machine learning models for regression and time series forecasting has been provided to predict revenues and applied on Microsoft's Finance organization real finance data [16]. The travel toll revenue forecasting has been applied and illustrated in a probability distribution, using a Neural Network model [17]. Moreover, an analysis on historical data has been conducted as well using a Neural Network model, in order to predict rental income for enterprises having several malls [18].

However, although several previous studies propose to identify future technology development, none of them rely on combining patents, publications and technologies revenues as key performance indicators. Accordingly, and based on our research, the current study has not been applied so far when evaluating and monitoring technologybased product development in this specific context.

\section{Research Design}

The analysis of patents, publications and revenues data is considered as a quantitative approach to assess their impact on the technology-based product success. Precisely, this study visualizes the historical growth of patents, publications and revenues dimensions, as well as their predictive future variation for a given candidate 
technology, using two separate types of Neural Networks for comparison purposes: a Wide and Deep Neural Network model and a Recurrent Neural Network model. These neural networks are to be implemented as following: The one-dimensional WDNN (ODWDNN) and RNN (ODRNN) models are employed to separately predict the patents and publications growth. Therefore, their predicted results, as well as the historical data of these two dimensions, will then be used as inputs in either a Multidimensional Wide and Deep Neural Network (MDWDNN) classifier for WDNN, or a Multidimensional Recurrent Neural Network (MDRNN) classifier for RNN. These multidimensional neural networks are designed to forecast future revenues ranges for specific trending technologies, and therefore evaluating and measuring technology-based product power.

Why use a Neural Network model: Based on different studies, Artificial Neural Network models can outperform traditional models, such as Linear Regression [19] and Autoregressive Integrated Moving Average [20] in the context of time series forecasting and obtaining higher accuracy. In addition, since this study model will eventually be targeting organizations in a future work, a predictive neural network can be trained and tested through historical Big Data for an unsupervised [21] and dynamic learning [22].

Why use a Wide and Deep Neural Network model: A wide and deep model can handle complex large-scale data, integrating heterogeneous input data [23]. Furthermore, Deep Learning algorithms can generalize the relationships in the data and the extracted representation, by extracting rare or new combinations by transitivity of correlations. However, deep neural network may over-generalize and extract less relevant features [24]. Accordingly, the exception rules and the memorization of features correlations or interactions in the historical data, is a crucial need to improve the neural network forecasting. Hence the power of combining a deep model for learning interactions within historical data and then generalizing the output on new data with a wide model for memorizing specific rules and learning exceptions [24].

Why use a Recurrent Neural Network model: A recurrent neural network can process sequences of inputs, which represent the time series of patents and publications in our case, with different lengths, to return a sequence of outputs [25]. In addition, LSTM (Long Short-Term Memory) can be used in the RNN to reduce the vanishing gradient problem that occurs in the basic recurrent neural networks [26].

\subsection{Proposed Methodology}

The current methodology relies on three dimensions: patents, publications and revenues. It first predicts the growth of the number of patents and the number of publications respectively, which will be used in the final prediction phase of revenues ranges. It covers different principle objectives explained in Table 1. Noting that these objectives and tasks are applied for both neural network types.

Table 1. Methodology design objectives.

\begin{tabular}{|l|l|}
\hline \multicolumn{2}{|c|}{ Objective: Data Collection } \\
\hline 1 & Listing new and old trending technologies based on different sources. \\
\hline 2 & Searching for related keywords for each technology. \\
\hline \multicolumn{2}{|c|}{ Objective: Database integration } \\
\hline 3 & Onserting the collected data into a new integrated database. \\
\hline \multicolumn{2}{|c|}{ Objective: Data Collection } \\
\hline 4 & $\begin{array}{l}\text { Extracting patents, publications and revenues data for each technology from several } \\
\text { sources based on keywords matching. }\end{array}$ \\
\hline & Objective: Database integration \\
\hline
\end{tabular}




\begin{tabular}{|c|c|}
\hline 5 & Manipulating and inserting the collected data into the integrated database. \\
\hline \multicolumn{2}{|r|}{ Objective: Data manipulation } \\
\hline 6 & Computing the total number of patents / publications per technology per year. \\
\hline \multicolumn{2}{|r|}{ Objective: Datasets creation } \\
\hline 7 & $\begin{array}{l}\text { Grouping patents / publications data by technology for } \max \text { years. } \max \text { represents the } \\
\text { maximum number of years per technology where historical data is available in the } \\
\text { training dataset. }\end{array}$ \\
\hline \multicolumn{2}{|r|}{ Objective: Neural Network 1 (NN1) implementation } \\
\hline 8 & Training the first Neural Network (ODWDNN and ODRNN) with patents data. \\
\hline 9 & Testing the Neural Network (ODWDNN and ODRNN) with patents data. \\
\hline 10 & Predicting the number of patents for the candidate technology for the next $p$ years. \\
\hline \multicolumn{2}{|r|}{ Objective: Database integration } \\
\hline 11 & $\begin{array}{l}\text { Inserting the output data of Neural Network } 1 \text { (ODWDNN and ODRNN) into the } \\
\text { integrated database. }\end{array}$ \\
\hline \multicolumn{2}{|r|}{ Objective: Neural Network 1 (NN1) implementation } \\
\hline 12 & Training the first Neural Network (ODWDNN and ODRNN) with publications data. \\
\hline 13 & Testing the Neural Network (ODWDNN and ODRNN) with publications data. \\
\hline 14 & $\begin{array}{l}\text { Predicting the number of publications for the candidate technology for the next } p \\
\text { years. }\end{array}$ \\
\hline \multicolumn{2}{|r|}{ Objective: Database integration } \\
\hline 15 & $\begin{array}{l}\text { Inserting the output data of Neural Network } 1 \text { (ODWDNN and ODRNN) into the } \\
\text { integrated database. }\end{array}$ \\
\hline \multicolumn{2}{|r|}{ Objective: Dataset creation } \\
\hline 16 & Grouping patents, publications and revenues data by technology and by year. \\
\hline \multicolumn{2}{|r|}{ Objective: Neural Network 2 (NN2) implementation } \\
\hline 17 & $\begin{array}{l}\text { Training the second Neural Network (MDWDNN and MDRNN) based on the three } \\
\text { dimensions. }\end{array}$ \\
\hline 18 & $\begin{array}{l}\text { Testing the Neural Network (MDWDNN and MDRNN) with patents, publications } \\
\text { and revenues data. }\end{array}$ \\
\hline 19 & Predicting the revenues classes for the candidate technology for future $p$ years. \\
\hline \multicolumn{2}{|r|}{ Objective: Database integration } \\
\hline 20 & $\begin{array}{l}\text { Inserting the output data of Neural Network } 2 \text { (MDWDNN and MDRNN) into the } \\
\text { integrated database. }\end{array}$ \\
\hline \multicolumn{2}{|r|}{ Objective: Results visualization and Decision-making } \\
\hline 21 & Illustrating the revenues variation in statistical graphs. \\
\hline 22 & Evaluating the candidate technology based on business perspective. \\
\hline
\end{tabular}

\subsection{Neural Network Structures}


The present Neural Networks are implemented using the TensorFlow ${ }^{1}$ software library under Python. As previously mentioned, two Neural Network types are structured as following:

Neural Network 1. The first neural network is implemented using two different models for comparison purposes, in order to separately estimate the number of patents and the number of publications.

One-dimensional WDNN (ODWDNN). This Neural Network is structured as a "DNN Linear Combined Regressor" where the predicted output represents a continuous variable. It is designed with the same architecture and configuration as the WDNN of the Saade, Jneid \& Saleh paper [10]: ODWDNN contains $h$ hidden layers, and each layer contains a specific number no of nodes. It is built based on the Adam optimization method which is designed for training deep neural networks and can outperform other stochastic optimization methods [27]. In addition, the number of patents / publications will be predicted for the technology in question for each future year separately, where each predicted output is serving as input for the next prediction until the number of future years $p$ is reached.

One-dimensional RNN (ODRNN). This Neural Network model is structured with the same architecture and configuration as the RNN in the study of Saade, Jneid \& Saleh [10]: ODRNN contains $h$ recurrent hidden layers, with a specific number no of nodes. It is built based on the following features:

- Long Short-Term Memory (LSTM) cells: they are activation cells that can outperform traditional recurrent layers.

- Sequence-to-Sequence (seq2seq) neuronal architecture: where the RNN encodes a variable-length sequence of inputs into a fixed-length vector representation and then decoding a fixed-length vector representation into a sequence of outputs [28]. Noting that the input sequences lengths vary according to the data availability of each technology.

- Many-to-many structure: where both the input and the output represent sequences of data with same or different lengths.

- Adam optimizer: can outperform other stochastic optimization methods [27].

In addition, the output layer represents a sequence of number of patents / publications forecasted in a single step from Year 1 till Yearp.

Neural Network 2. The second Neural Network is implemented using two different models for comparison purposes. They are designed as multidimensional neural networks and classification predictive models. Patents and publications data represent the inputs dimensions and the ranges of revenues represent the output classes. This classifier would return a probability distribution for each of the defined classes.

Multi-dimensional WDNN (MDWDNN). MDWDNN is structured as a "DNN Linear Combined Classifier". Adam is applied as well in the MDWDNN as an optimization method. This Neural Network consists of the following layers:

\footnotetext{
${ }^{1}$ Tensorflow is an open-source API created by Google and is used for Machine Learning purposes.
} 


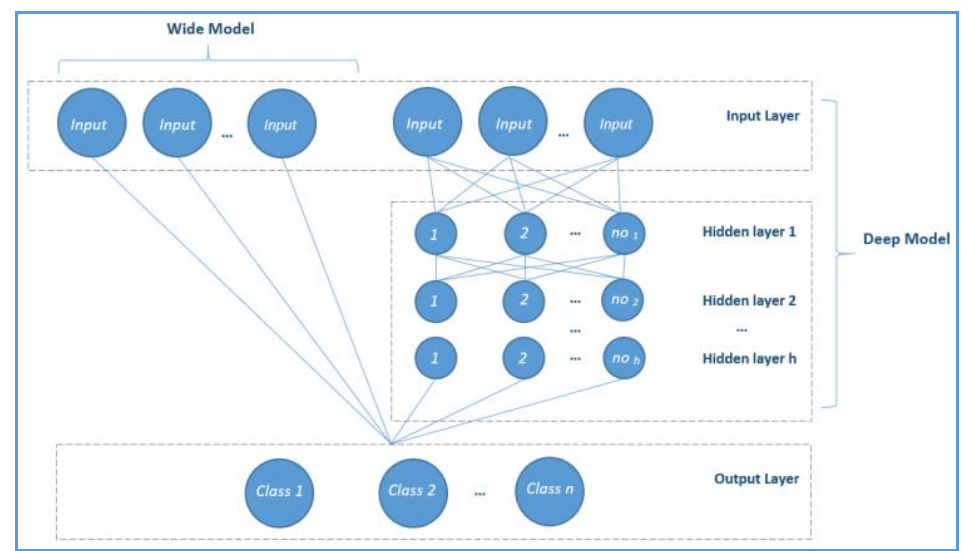

Fig. 1. Multi-dimensional Wide and Deep Neural Network (MDWDNN).

- Input Layer: The input layer represents the number of patents and publications for a technology for each available year.

- Hidden Layers: This neural network contains $h$ hidden layers, and each layer contains no nodes.

- Output layer: represents the revenues ranges to be forecasted. Noting that each predicted output is serving as input for the next prediction until the number of future years $p$ is reached.

Multi-dimensional RNN (MDRNN). MDRNN processes time series sequences of multidimensional inputs, and predicts the probability distribution for each class for these sequences. In addition, it is based on encoder-decoder sub-models using LSTM and is structured as a Many-to-One model for classification usage, where the inputs represent sequences of data and the output represents a category. Moreover, Adam is applied as well in the MDRNN as an optimization method. Accordingly, it is built based on the following three layers:

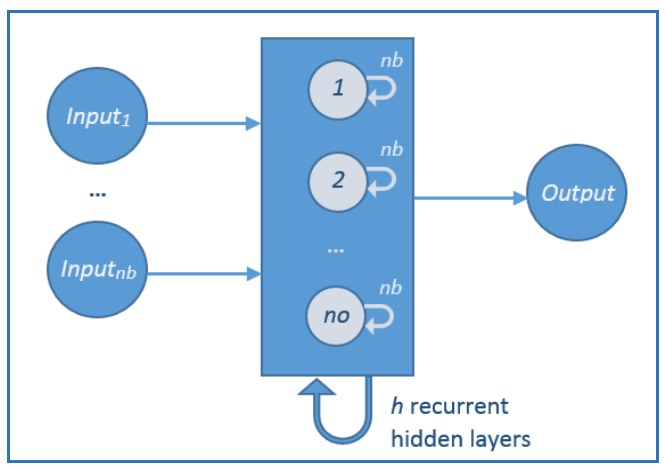

Fig. 2. Multi-dimensional Recurrent Neural Network (MDRNN).

- Input Layers: MDRNN consists of a specific number $n b$ of input layers or dimensions. They take time series sequences with a variable length.

- Hidden Layers: Since a multidimensional recurrent neural network (MDRNN) consists of replacing the single self-connection in each node in a standard recurrent neural network with as many of self-connections as there are dimensions in the input data [29], MDRNN contains $h$ recurrent hidden layers, with a specific number $n o$ of nodes, where each node in each hidden layer contains $n b$ self-connections.

- Output Layer: The output layer represents a sequence of classes to be predicted in a single step from Year till Year $_{\mathrm{p}}$.

\section{Experimentation}


In order to train and test the neural networks, patents, publications and revenues data are collected for 15 trending technologies. Noting that since this study model will be targeting actual organizations in a future work, this predictive neural network can be eventually trained and tested using historical Big Data. The candidate technology's data are applied on the neural networks to predict its future patents, publications and its revenues ranges for the next four years.

\subsection{Data Collection}

Technologies Listing. Different web sources such as IEEE, Scientific American, Elsevier, etc. are used to list several trending technologies and their related keywords, such as the "Additive manufacturing" term for the "3D printing" technology.

Patent Data Source. Data of the granted patents applications that have been published before December 31, 2019, are extracted from the United States Patent and Trademark Office (USPTO) database which is considered among the richest intellectual property databases [30].

Publications Data Source. Publications data such as title, year, author name, etc. are extracted from Springer that includes a wide range of bibliometric resources.

Revenue Data Source. The revenues related to each technology for several years have been extracted from different web sources, such as: Statista web pages, Smart Insights and different reports and articles.

\subsection{Training, Testing and Prediction Datasets Creation}

Given that the amount of the collected data is limited in volume, it has been split manually into training and testing datasets. Furthermore, in order to compare the predicted values with the actual values and to visualize the accuracy of the Neural Networks, the validation dataset is also taken as the prediction dataset, where "Virtual Reality" represents the candidate technology for which its future values will be forecasted. In addition, the predicted outputs of ODWDNN and ODRNN are integrated into MDWDNN and MDRNN respectively, along with the historical data of patents, publications and revenues dimensions. Furthermore, given that Neural Network 2 (MDWDNN and MDRNN) represents a classifier model that predicts the future revenues ranges for a specific trending technology, revenues data have been grouped into 3 classes based on data availability, as following:

Table 2. Revenues classes.

\begin{tabular}{|l|l|}
\hline Class & Revenues ranges (in USD) \\
\hline 1 & $0<=$ Revenue $<=10$ Billion \\
\hline 2 & 10 Billion $<=$ Revenue $<=50$ Billion \\
\hline 3 & Revenue $>50$ Billion \\
\hline
\end{tabular}

\subsection{Neural Networks Implementation}

The configurations and the training parameters of the neural networks have been adjusted and tuned progressively and determined experimentally according to the most accurate results. In addition, in order to ensure a fair comparison, the number of training steps applied for both neural network types are similar, and equal to 1000 steps. 
Neural Network 1. Regarding the results accuracy of the ODWDNN and ODRNN models, it is calculated based on the same steps and formulae employed in the Saade, Jneid \& Saleh article [10].

One-dimensional WDNN (ODWDNN). ODWDNN is implemented with the following main parameters, as defined in the Proposed Methodology section:

$p=4$ years; $\max =10$ years; $h=5$ hidden layers; $n o l=1000$ nodes; $n o_{2}=750$ nodes; nо $3=500$ nodes; no $4=300$ nodes; no $5=150$ nodes

One-dimensional RNN (ODRNN). It represents a Sequence-to-Sequence Recurrent Neural Network. It has been built and implemented based on the following main parameters defined in the previous sections:

$p=4$ years; $\max =10$ years; $h=2$ recurrent layers; $n o=250$ hidden nodes per layer

Neural Network 2. The results accuracy of the Multi-dimensional Neural Networks Classifiers MDWDNN and MDRNN are generated based on the built-in Softmax function that provides the probability distribution for output classes [31], which means Softmax takes the predicted output values and normalizes them into probabilities.

Multi-dimensional WDNN (MDWDNN). MDWDNN is implemented with the following main parameters, as defined in the Proposed Methodology section:

$p=4$ years; $\max =10$ years; $h=5$ hidden layers; $n o_{1}=1000$ nodes; $n o_{2}=750$ nodes;

no $3=500$ nodes; no $4=300$ nodes; no $5=150$ nodes

Multi-dimensional RNN (MDRNN). The parameters of MDRNN are configured as following:

$n b=2 ; p=4$ years; $h=2$ recurrent layers; $n o=250$ hidden nodes per layer

\subsection{Results}

The following subsections represent the obtained results for the "Virtual reality" candidate technology using both types of Neural Networks, illustrating the predictions quality, where the ordinate axes represent the dimensions values and the abscissa axes represent the time.

\section{Neural Network 1}

As per the below graphs and Table 3, the ODRNN achieves a better performance and accuracy and outperforms the ODWDNN, given that the average prediction accuracy of the ODRNN is higher than that of the ODWDNN.

Table 3. Prediction accuracies of Neural Networks 1.

\begin{tabular}{|l|l|l|l|}
\hline \multicolumn{2}{|c|}{ ODWDNN } & \multicolumn{2}{c|}{ ODRNN } \\
\hline $\begin{array}{l}\text { Patents prediction } \\
\text { accuracy }\end{array}$ & $\begin{array}{l}\text { Publications } \\
\text { Prediction accuracy }\end{array}$ & $\begin{array}{l}\text { Patents prediction } \\
\text { accuracy }\end{array}$ & $\begin{array}{l}\text { Publications } \\
\text { Prediction accuracy }\end{array}$ \\
\hline $51.40 \%$ & $83.58 \%$ & $84.86 \%$ & $70.18 \%$ \\
\hline Average prediction accuracy: 67.49\% & Average prediction accuracy: 77.52\% \\
\hline
\end{tabular}


One-dimensional WDNN (ODWDNN).

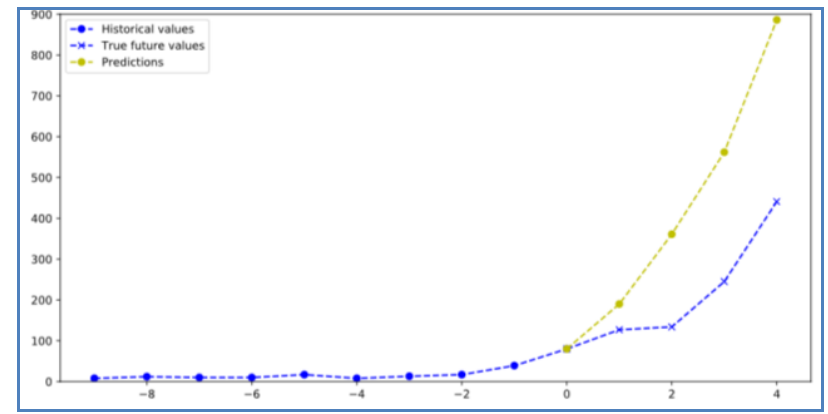

Fig. 3. Actual and predicted number of patents in the ODWDNN for the "Virtual reality" technology from 2006 to 2019.

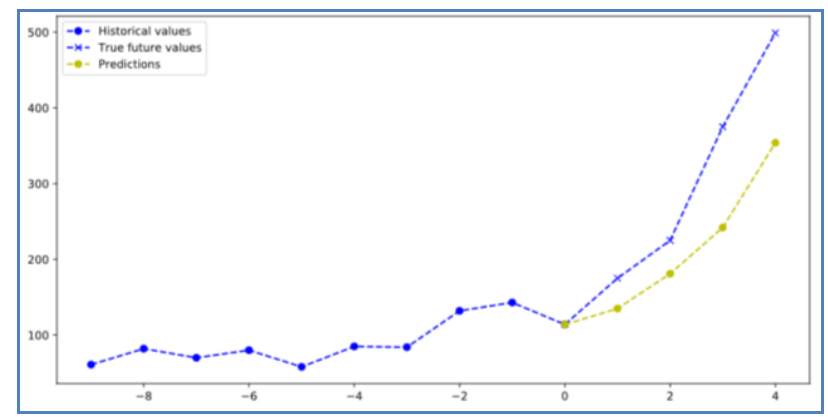

Fig. 4. Actual and predicted number of publications in the ODWDNN for the "Virtual reality" technology from 2006 to 2019.

One-dimensional RNN (ODRNN).

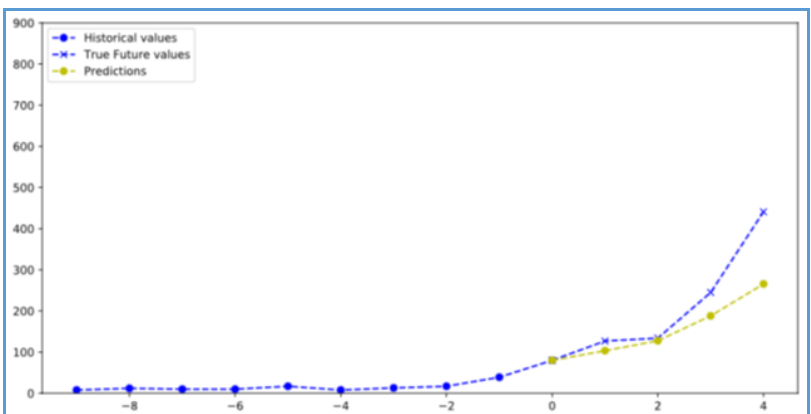

Fig. 5. Actual and predicted number of patents in the ODRNN for the "Virtual reality" technology from 2006 to 2019.



Fig. 6. Actual and predicted number of publications in the ODRNN for the "Virtual reality" technology from 2006 to 2019. 
Neural Network 2. As per the following table, the MDRNN achieves a better performance and accuracy and outperforms the MDWDNN, given that the MDRNN was able to detect the actual revenue class of the fourth year by $39.94 \%$, whereas the fourth probability of the MDWDNN was $0 \%$.

Table 4. Prediction accuracies of Neural Network 2.

\begin{tabular}{|l|l|l|l|l|l|l|}
\hline & \multicolumn{3}{|c|}{ MDWDN } & \multicolumn{3}{c|}{ MDRNN } \\
\hline $\begin{array}{c}\text { Future } \\
\text { years }\end{array}$ & $\begin{array}{l}\text { Actual } \\
\text { Class }\end{array}$ & $\begin{array}{l}\text { Predicted } \\
\text { Class }\end{array}$ & $\begin{array}{l}\text { Actual Class } \\
\text { Prediction } \\
\text { Accuracy }\end{array}$ & $\begin{array}{l}\text { Actual } \\
\text { Class }\end{array}$ & $\begin{array}{l}\text { Predicted } \\
\text { Class }\end{array}$ & $\begin{array}{l}\text { Actual Class } \\
\text { Prediction } \\
\text { Accuracy }\end{array}$ \\
\hline Year 1 & 1 & 1 & $99.50 \%$ & 1 & 1 & $100 \%$ \\
\hline Year 2 & 1 & 1 & $99.92 \%$ & 1 & 1 & $99.99 \%$ \\
\hline Year 3 & 1 & 1 & $99.99 \%$ & 1 & 1 & $99.99 \%$ \\
\hline Year 4 & 2 & 1 & $0 \%$ & 2 & 1 & $39.94 \%$ \\
\hline & $\begin{array}{l}\text { Average prediction accuracy: } \\
74.85 \%\end{array}$ & $\begin{array}{l}\text { Average prediction accuracy: } \\
8.98 \%\end{array}$ \\
\hline
\end{tabular}

\section{Conclusion/Discussion}

Research and Development-based digital innovation activities represent a main driver for new product development and an added value in today's business economy. However, despite the fact that organizations are constantly developing new products while increasing the R\&D investments and deploying new technologies, innovative trends are prone to failure. In addition, when evaluating a product life-cycle, the decision-makers of the organization first need to consider the potential disruptive innovation. Accordingly, predicting technology-based product success is a crucial need and a prerequisite step before proceeding with investments. A new quantitative approach has been proposed in the current paper, based on patents, publications and revenues dimensions analysis, and using Neural Network models that can evaluate and monitor a candidate technology-based product development. These dimensions are considered major key performance indicators to measure the technology power. Precisely, this paper covers two main steps: forecasting patents and publications growths separately using a common predictive Neural Network model, then integrating the results into a Multi-dimensional Neural Network classifier model in order to predict the future revenue-volume which can reflect the technology-based product success. The present methodology is applied using two different types of Neural Network models for comparison purpose: Wide and Deep Neural Networks and Recurrent Neural Networks. These neural networks have been implemented using United States Patent and Trademark Office data, Springer publications data and several web sources in order to collect technologies revenues data. In addition, this method has been experimented on 15 trending technologies to train the neural networks, then applied on one additional candidate technology, "Virtual reality", for the testing and prediction phases. The findings show that the Recurrent Neural Networks models achieve higher average prediction accuracy, and outperform the Wide and Deep Neural Networks in both steps, proving to be a valid model that can enhance digital innovation development.

Therefore, the proposed approach provides answers related to technology-based product success and reliable prediction models. The latter can be used by decisionmakers in the organizations to evaluate and monitor a new trending innovation, in order to either measure its impact on existing product market performance, or to adopt this potential disruptive technology that may lead to a new product development.

Consequently, addressing this estimation is a crucial prerequisite step before proceeding with investments, where organizations can improve decision making in innovative technology-based product development. Furthermore, this method can be applied on several candidate technologies in order to prioritize and identify the most appropriate ones for a given investment project.

This methodology can be further developed by addressing its current limitations. For instance, accessing Big data represents the most challenging task faced in the current study where patents data are based uniquely on USPTO patents applications and 
publications information are extracted only from Springer. Moreover, the proposed methodology can be further evolved to include products composed of multiple major technologies to support all types of technologies-based products.

\section{References}

1. Distanont, A. and Khongmalai, O., 2018. The role of innovation in creating a competitive advantage. Kasetsart Journal of Social Sciences.

2. Jneid, M. and Saleh, I., 2015. Improving start-ups competitiveness and innovation performance: the case of Lebanon. In ISPIM Conference Proceedings (p. 1). The International Society for Professional Innovation Management (ISPIM).

3. Christensen, C. M., 2013. The innovator's dilemma: when new technologies cause great firms to fail. s.1.:Harvard Business Review Press.

4. Sun, J., Gao, J., Yang, B.and Tan, R., 2008, September. Achieving disruptive innovationforecasting potential technologies based upon technical system evolution by TRIZ. In 2008 4th IEEE International Conference on Management of Innovation and Technology (pp. 18-22). IEEE.

5. Sheffi, Y., 2015. The power of resilience: How the best companies manage the unexpected. mit Press.

6. Obal M., Ibrahim S. (2016) An Investigation on the Role of Disruptive Technology Adoption on New Product Market Performance and Launch Timeliness. In: Obal M., Krey N., Bushardt C. (eds) Let's Get Engaged! Crossing the Threshold of Marketing's Engagement Era. Developments in Marketing Science: Proceedings of the Academy of Marketing Science. Springer, Cham.

7. Nagy, D., Schuessler, J. \& Dubinsky, A., 2016. Defining and identifying disruptive innovations. Industrial Marketing Management, Volume 57, pp. 119-126.

8. Momeni, A. \& Rost, K., 2016. Identification and monitoring of possible disruptive technologies by patent-development paths and topic modeling. Technological Forecasting \& Social Change, Volume 104, pp. 16-29.

9. Shang, T., Miao, X. \& Abdul, W., 2019. A historical review and bibliometric analysis of disruptive innovation. International Journal of Innovation Science.

10. Saade, M., Jneid, M., \& Saleh, I., 2019. Predicting technology success based on patent data, using a wide and deep neural network and a recurrent neural network. In IBIMA 33 Conference proceedings. IBIMA.

11. Geum, Y., Lee, S., Yoon, B. and Park, Y., 2013. Identifying and evaluating strategic partners for collaborative R\&D: Index-based approach using patents and publications. Technovation, 33(6-7), pp.211-224.

12. Han, F. and Magee, C.L., 2018. Testing the science/technology relationship by analysis of patent citations of scientific papers after decomposition of both science and technology. Scientometrics, 116(2), pp.767-796.

13. Wisła, R., 2017. Patent Data in Economic Analysis. Intellectual Property Rights, p.65.

14. Daim, T.H., Rueda, G., Martin, H. and Gerdsri, P., 2018. Forecasting Emerging Technologies: Use of Bibliometrics and Patent Analysis. World Scientific Series in $R \& D$ Management, p.305.

15. Wang, B., Liu, Y., Zhou, Y. and Wen, Z., 2018. Emerging nanogenerator technology in China: A review and forecast using integrating bibliometrics, patent analysis and technology roadmapping methods. Nano energy, 46, pp.322-330.

16. Gajewar, A., \& Bansal, G. (2016). Revenue Forecasting for Enterprise Products. arXiv preprint, arXiv:1701.06624.

17. Zhao, Y. \& Zhao, H., 2017. Evaluating Toll Revenue Uncertainty Using Neural Network Models. Shanghai, ScienceDirect, pp. 2949-2956.

18. Sanjaya, C., liana, M. \& Widodo, A., 2010. Revenue Prediction Using Artificial Neural Network. s.l., IEEE, pp. 97-99.

19. Veerachai Gosasang, W. C. S. K., 2011. A Comparison of Traditional and Neural Networks Forecasting Techniques for Container Throughput at Bangkok Port. The Asian Journal of Shipping and Logistics, December, 27(3), pp. 463-482.

20. Adebiyi, A. A., Adewumi, A. O. \& Ayo, C. K., 2014. Comparison of ARIMA and Artificial Neural Networks Models for Stock Price Prediction. Journal of Applied Mathematics, Volume 2014, p. 7.

21. Schmidhuber, J., 2015. Deep Learning in Neural Networks: An Overview. Neural Networks, Volume 61, pp. 85-117.

22. Alsheikh, M. A., Lin, S., Niyato, D. \& Tan, a. H.-P., 2014. Machine learning in wireless sensor networks:Algorithms, strategies, and applications. IEEE Communications Surveys and Tutorials, Volume 16(4), pp. 1996-2018. 
23. Elaraby, N., Elmogy, M. \& Barakat, S., 2016. Deep Learning: Effective Tool for Big Data Analytics. International Journal of Computer Science Engineering (IJCSE), Volume 5, pp. 254-262.

24. Cheng, H.-T.et al., 2016. Wide \& Deep Learning for Recommender Systems, s.1.: Google.

25. Zhou, S.K., Rueckert, D. and Fichtinger, G. eds., 2019. Handbook of medical image computing and computer assisted intervention. Academic Press.

26. Goyal, P., Pandey, S. and Jain, K., 2018. Deep learning for natural language processing. Deep Learning for Natural Language Processing: Creating Neural Networks with Python [Berkeley, CA]: Apress, pp.138-143.

27. Kingma, D.P. and Ba, J., 2014. Adam: A method for stochastic optimization. arXiv preprint arXiv: 1412.6980.

28. Sutskever, I., Vinyals, O. and Le, Q.V., 2014. Sequence to sequence learning with neural networks. In Advances in neural information processing systems, pp. 3104-3112.

29. Graves, A., Fernández, S. \& Schmidhuber, J., 2007. Multidimensional Recurrent Neural Networks. Berlin, Heidelberg, Springer, pp. 549-558.

30. USPTO. (2018, June). USPTO. Retrieved from https://www.uspto.gov

31. Tang, D., Qin, B. and Liu, T., 2015, September. Document modeling with gated recurrent neural network for sentiment classification. In Proceedings of the 2015 conference on Empirical methods in natural language processing (pp. 1422-1432). 